\title{
Perceiving action boundaries: Learning effects in perceiving maximum jumping-reach affordances
}

\author{
Verónica C. Ramenzoni, Tehran J. Davis, Michael A. Riley, and Kevin Shockley \\ University of Cincinnati, Cincinnati, Ohio
}

\begin{abstract}
Coordinating with another person requires that one can perceive what the other is capable of doing. This ability often benefits from opportunities to practice and learn. Two experiments were conducted in which we investigated perceptual learning in the context of perceiving the maximum height to which an actor could jump to reach an object. Those estimates were compared with estimates that perceivers made for themselves. In Experiment 1, participants initially underestimated the maximum jumping-reach height both for themselves and for the actor. Over time, without explicit feedback, the participants were able to improve estimates of their own maximum jumping-reach height, but estimates for the actor did not improve. In Experiment 2, participants observed the actor perform either an action related but nonidentical to jumping (lifting a weight by squatting) or a nonrelated activity (rotating the torso). The participants who observed the actor perform the related action were able to improve the accuracy of their perceptual reports for the actor's maximum jumping-reach height, but the participants who watched the actor perform the nonrelated task were unable to do so. The results indicate some degree of independence between perceived affordances for the self and others, suggesting that affordance judgments are not entirely dependent on or determined by characteristics of the perceiver.
\end{abstract}

Most human behavior takes place within a social environment. Social coordination of behavior can be achieved by selecting and controlling one's actions with regard to what other actors are doing, are about to do, or are potentially capable of doing. For example, if an actor knows that a companion cannot lift a heavy object, the actor knows to provide assistance and, furthermore, can determine how much lifting force he or she must contribute so that the pair can lift the object together. Such instances of social coordination often depend critically on each actor's ability to perceive and anticipate the actions of others. The same is true when an agent's goal is to merely observe the behaviors of another agent, rather than to actively coordinate with the other agent, such as when a caregiver minds a child who is playing alone.

One perspective on social perception-action emphasizes the role of embodied simulation processes (motor resonance in the mirror neuron system) that are hypothesized to enable observers to interpret and predict others' actions via the observers' own neural systems for movement (e.g., Gallese, 2005; see also Sebanz, Bekkering, \& Knoblich, 2006). A second perspective emphasizes the ability to perceive affordances (opportunities for action; J. J. Gibson, 1979) for other actors (Ramenzoni, Riley, Shockley, \& Davis, 2008b; see also Fischer, 2003; Lamm, Fischer, \& Decety, 2007). Although motivated by different theoretical assumptions, these two perspectives are not exclusive, and they may reflect complementary aspects of social perception-action (Knoblich \& Sebanz, 2008). As was noted by Ramenzoni et al. (2008b), simulations cannot be absolutely indeterminate or agent neutral (Pacherie \& Dokic, 2006; cf. Gallese, 2005) or observers would usually fare rather poorly at predicting or understanding actions that require a tight metrical fit between another agent and the environment. Any cognitive processes related to predicting or understanding the actions of an agent thus need to have access to situated, context-sensitive information about the agent's action capabilities in a given environmental setting (i.e., what behaviors are afforded that agent by the environment). By this view, broad theoretical progress in social perception-action may depend on developing a more thorough understanding of social affordances.

Accordingly, in the present work, we did not measure or manipulate motor resonance in the mirror neuron system or any other neural processes believed to be directly involved in embodied simulation, but instead, we focused on some unresolved issues regarding the perception of affordances for others. It has been shown that in some cases, perceivers can prospectively estimate what is afforded an agent with a high degree of accuracy (Mark, 2007; Ramenzoni, Riley, Davis, Shockley, \& Armstrong, 2008; Ramenzoni, Riley, Davis, \& Snyder, 2005; Ramenzoni, Riley, Shockley, \& Davis, 2008a, 2008b; Stoffregen, Gorday, Sheng, \& Flynn, 1999; Weast, 2009). Perceivers are sensitive to both body-scaled affordances, defined by the geometric fit between the actor and the environment, and action-scaled affordances, defined by dynamic properties

M. A. Riley, michael.riley@uc.edu 
of the actor (i.e., force generation, acceleration, etc.) in relation to the environment, for another person. However, it was often the case in many of the studies cited above that perceivers were less accurate at perceiving affordances for others than for themselves. It is not known whether this is due to the perceivers' initially basing their estimates of what is afforded an agent on what is afforded themselves, nor is it known whether perceivers can learn to more accurately perceive affordances for another. This study was directed at those two issues.

\section{Do Perceived Affordances for Others Depend on the Self?}

Ramenzoni et al. (2008) found that after watching an actor walk while the actor wore ankle weights that reduced his or her maximum jumping-reach height, observers who were not explicitly aware of the presence of the weights provided appropriately lower perceptual category boundaries (i.e., the boundary separating what could be reached by a vertical jump and what was out of reach) than they provided for the actor when he or she was unencumbered. The result indicates that observers are often quite sensitive to the action capabilities of others or at least to changes in action capabilities. A follow-up study (Ramenzoni et al., 2008a) showed that perception of the maximum jumping-reach height affordance for another person was also influenced by changes in the observer's own action capabilities. When ankle weights were attached to the $o b$ server (reducing the observer's own maximum jumping height), the observer's estimates of an unencumbered actor's jumping height were lower than estimates obtained by observers who were not wearing ankle weights. This finding suggests that the observer's own action capabilities influence perception of the limits of another person's action capabilities.

Although perceptual reports of what is afforded an actor are not completely determined by the observer's own action capabilities (cf. Gallese, 2005) - observers can distinguish what actors with different abilities can do - they are not completely independent of the observer, either. The dependence between the perceiver's own action capabilities and estimates of affordances for another person might, however, diminish with perceptual experience and learning. It is critical to characterize any such changes, because the presence or absence of learning effects will need to be accounted for by any model of social perceptionaction, whether inspired by embodied simulation, ecological psychology, or some hybrid of the two.

\section{Learning to Perceive Affordances}

Changes in affordance judgments might arise through perceptual attunement or calibration (e.g., Fajen, 2005b; Fajen \& Devaney, 2006; Hove, Riley, \& Shockley, 2006). Attunement involves a change in the informational variable(s) selected as the basis for an action or perceptual response (E. J. Gibson, 1991; J. J. Gibson, 1966; Jacobs \& Michaels, 2006), whereas calibration refers to the scaling of actions or perceptual reports with reference to a given level of the detected informational variable (Fajen,
$2005 \mathrm{a}, 2005 \mathrm{c})$. It is often difficult to distinguish these processes when documenting learning effects, and they are not exclusive - both can occur to varying degrees and at varying points in the learning process.

Prior research has shown that perceivers can refine their estimates of another person's maximum jumping-reach height if they are able to observe the person performing a related but nonidentical action, such as walking, because that activity is informative about the actor's capabilities (Ramenzoni et al., 2008). Even without introducing new potential sources of information, providing a perceiver with repeated opportunities to sample the optic array might allow the perceiver to extract new information that may have previously gone undetected (Mark, Balliett, Craver, Douglas, \& Fox, 1990). Mark et al. (see also Stoffregen, Yang, \& Bardy, 2005) found that movement associated with naturally occurring postural sway provided perceivers with new opportunities to extract information to support fine-tuning of affordance judgments when the perceivers' action capabilities had been modified. This fine-tuning may have reflected attunement, calibration, or both.

\section{The Present Study}

In previous studies, we found that although perceivers are fairly accurate at perceiving maximum jumping-reach height affordances for themselves and an actor, some degree of error - typically, underestimation on the order of 10-15 cm (e.g., Ramenzoni et al., 2008) —was observed. This indicates room for improvement that could be achieved through learning. In the present study, we used procedures similar to those of Ramenzoni et al. (2008) to investigate processes of perceptual learning related to the perception of affordances for an actor. In Experiment 1 , we determined whether estimates of the maximum height one could reach by jumping improved in accuracy over time (i.e., with repeated opportunities to sample the optic array and to provide perceptual reports). Observers repeatedly estimated this affordance boundary for both themselves and an actor. A parallel pattern of change for self and actor affordance judgments could suggest that perception of another person's actions is dependent on one's own action capabilities. In contrast, different patterns of change over time, such as an apparent learning effect for self-estimates but not for actor estimates, might suggest that dependence of actor estimates on one's own action capabilities, if it occurs, may be limited to serving as an initial basis for ballpark estimates of an unfamiliar actor's abilities. In Experiment 2, we determined whether watching an actor perform an action that required the generation of vertically directed force with the legs would lead to an improvement in perceivers' estimates of the actor's maximum jumping-reach height.

\section{EXPERIMENT 1}

Feedback about perceptual reports can serve to guide perceptual learning so that observers can correctly identify a specifying variable (i.e., a perceptual variable that maps 
unambiguously to the property that the observer intends to perceive) and can properly calibrate their responses (e.g., Withagen \& Michaels, 2005). However, Mark et al. (1990) showed that even in the absence of explicit feedback, perceivers are capable of refining their estimates of what is afforded. In that study, participants were asked to make judgments relative to the sit-on-ability of a chair (the extent to which it could be sat on). The participants' sitting ability (i.e., the maximum height of a chair upon which they could sit) was manipulated by having them wear $10-\mathrm{cm}$ blocks on their feet, effectively increasing each person's maximum sitting height by $10 \mathrm{~cm}$. The participants could improve their judgments of sit-on-ability (they could account for the change in action capabilities resulting from the attached blocks) even in the absence of practice or explicit feedback, as long as they were given the opportunity to make basic movements, including postural sway. When postural sway was reduced and the participants were forced to remain stationary, no such improvement took place. These findings were replicated by Stoffregen et al. (2005), who furthermore identified changes in postural sway that were directly tied to successful perceptual learning. Postural sway may have served an exploratory function in those studies, generating perceptual information that revealed invariants characterizing the observer's situated relation to the environment. Perceptual exploration of this kind can provide opportunities for perceptual learning (attunement or calibration) when explicit feedback is unavailable (Withagen \& Michaels, 2005). The main goal of Experiment 1 was to determine whether improved accuracy in perception of an affordance (i.e., perceptual learning) that occurs when making estimates for oneself (Mark et al., 1990) is likewise seen for similar affordance judgments for another, when feedback is not provided to the observer. This permitted an examination of the relative dependence (or independence) of affordance estimates for an actor on the observer's own perceived action capabilities.

\section{Method}

Participants. Twenty participants ranging in height from 151 to $187 \mathrm{~cm}(M=169.5 \pm 11.5 \mathrm{~cm})$ and in maximum jumping-reach height from 209.5 to $294 \mathrm{~cm}(M=248.1 \pm 24.95 \mathrm{~cm})$ reported to the laboratory in pairs. There was a significant positive correlation between participant height and maximum jumping-reach height $(r=.78, p<.05)$, as well as a modest yet significant correlation $(r=.48, p<.05)$ between height and vertical jump (i.e., maximum jumping-reach height - reaching height). The participants were not assigned to pairs on the basis of any criterion, nor were they randomly assigned by the experimenter; pair membership was determined by the participants' choosing the same experiment time slots. The paired participants differed in height by, on average, $21.10 \pm$ $7.13 \mathrm{~cm}$ (range $=11.5-35 \mathrm{~cm}$ ), and differed in maximum jumpingreach height by, on average, $38.85 \pm 15.90 \mathrm{~cm}($ range $=11-62 \mathrm{~cm})$. On a given trial, each member of the pair was assigned the role of either observer or actor. The participants swapped roles midway through the experimental session.

Apparatus. The observers were asked to make estimates of the limits of their own maximum vertical jumping reach or that of the actor when jumping to grasp a small $(5 \times 4 \mathrm{~cm})$ cylindrical object suspended from the ceiling. The cylinder was suspended in front of a uniform black wall and could be raised or lowered via a pul-

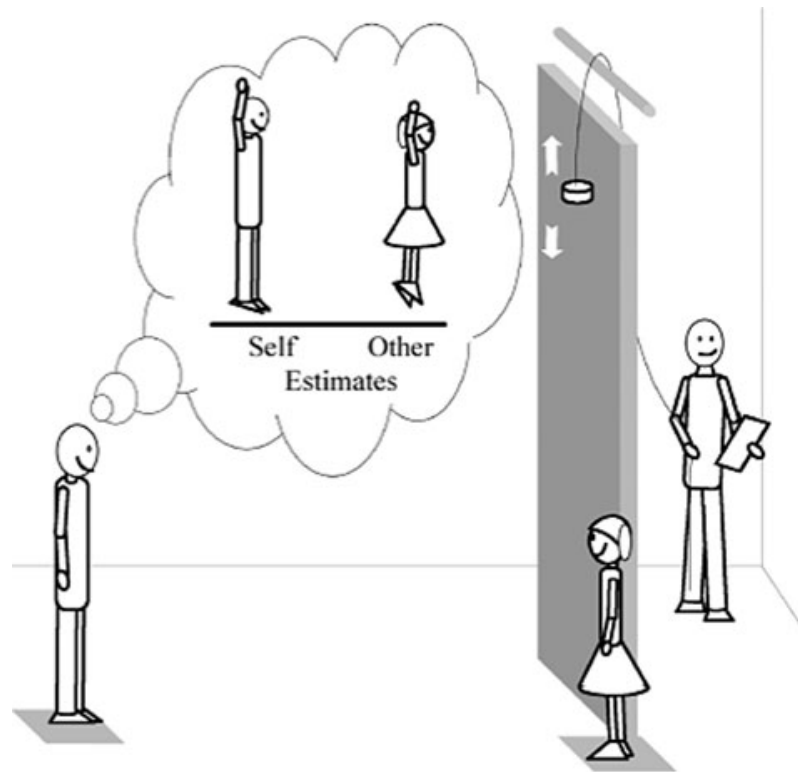

Figure 1. Apparatus. Participants directed an experimenter to raise or lower the small cylinder until they felt that it was just within reach if they or the actor (depending on experimental condition) were to jump from a standing position to reach it.

ley within a vertical range of $150-302 \mathrm{~cm}$ from the ground (see Figure 1).

Procedure. Maximum jumping-reach height was defined as the maximum height at which the cylinder could be grasped while performing a vertical jump (i.e., no steps were allowed) without making preparatory arm swings prior to the jump to create momentum, and with the preferred arm extended overhead. This definition was provided to the participants prior to beginning the experiment, but the participants did not perform the actual jumping task until all trials had been completed. The participants therefore did not see the other member of the pair perform the task until the experiment was over, although they did have opportunities to observe each other walking around the laboratory during the experiment before data collection occurred (cf. Ramenzoni et al., 2008). At the conclusion of the experimental trials, each participant performed the jumpingreach task. Jumps were made to grasp the same object for which estimates were made, in front of the same experimental apparatus. The first jump was made with the object at the average height that the participants had estimated for themselves. Depending on whether the participants could reach the object while jumping, the height of the object was varied over three subsequent trials. Each participant's maximum jumping-reach height was determined as the greatest of the three attempts.

In all conditions, the observers stood $3 \mathrm{~m}$ from the wall that served as the backdrop for the target cylinder. The actor was stationed directly beside the apparatus (from the observer's perspective, to the right side) at all times, allowing the observer to view both the actor and the apparatus concurrently. Both the observer and the actor were instructed to stand in place with their arms at their sides for the duration of the experiment. The actor was blindfolded and wore headphones playing music to prevent influence by hearing the perceptual estimates given by the observer. ${ }^{1}$

Perceptual reports were obtained using the method of adjustments (Guilford, 1954). The participants verbally instructed an experimenter who, while standing out of sight behind the apparatus, alternately raised or lowered the object by means of a rope and pulley until the observer indicated that the object was just at the perceived maximum jumping-reach height for the observer or for 
the actor, depending on the condition. The observers were allowed to fine-tune their responses during a given trial until they were satisfied. Between trials, the observers closed their eyes while the experimenter reset the apparatus. Successive ascending-descending estimates were averaged as 1 trial. Each participant completed 10 such trials for self-estimates and 10 trials for actor estimates, with self and actor trials presented in alternating blocks of 2 trials. Block order was counterbalanced across participants. When all of the trials were completed for the first participant, the members of the pair switched roles.

\section{Results and Discussion}

Averaged across trials, the participants were fairly accurate overall but exhibited a tendency to underestimate maximum jumping-reach height for themselves (mean estimate $=237.04 \pm 27.95 \mathrm{~cm}$; mean error $=$ mean estimate - actual jumping-reach height $=-11.19 \pm$ $33.40 \mathrm{~cm}$ ) and for the actor (mean estimate $=234.23 \pm$ $15.44 \mathrm{~cm}$; mean error $=-13.99 \pm 27.79 \mathrm{~cm})$. The initial self and actor responses differed, on average, by less than $0.05 \mathrm{~cm}$.

To determine the stability of the self-estimates and actor estimates over time, an ANOVA on perceived maximum jumping-reach height was conducted with judgment type (self vs. actor) and trial (Trials 1-10) as within-subjects factors. ${ }^{2}$ The analysis revealed a significant main effect of trial $\left[F(9,171)=3.12, p<.05, \eta_{\mathrm{p}}^{2}=.14\right]$. The judgment type $\times$ trial interaction was also significant $[F(9,171)=$ $3.03, p<.05$ ] (see Figure 2) and was as strong as the trial main effect $\left(\eta_{\mathrm{p}}^{2}=.14\right)$. Follow-up ANOVAs revealed a significant effect of trial for self-estimates $[F(9,171)=5.65$, $\left.p<.05, \eta_{\mathrm{p}}^{2}=.23\right]$ but not for actor estimates $(p>.05)$. The estimates increased and became more accurate over time for the observer, but estimates for the actor remained constant over trials.

We also examined patterns of correlation between the perceptual estimates (for both the observer and the actor) and the participants' height and jumping-reach height for Trials 1 and 10 . The participant height and self-estimates were significantly correlated for Trial $1(r=.80, p<.05)$ and Trial $10(r=.73, p<.05)$, as were the self-estimates and maximum jumping-reach height for Trial $1(r=.91$, $p<.05)$ and Trial $10(r=.91, p<.05)$. For Trial 10 only, the correlation of self-estimates with maximum jumpingreach height was significantly stronger than the correlation of self-estimates with participant height $[t(17)=$ $-2.52, p<.05]$. For the actor estimates, the estimates in Trial 10 (but not Trial 1) were significantly correlated with the actor's maximum jumping-reach height $(r=.55$, $p<.05$ ).

Hierarchical regressions were also employed to statistically control for the significant correlation between the participants' height and their jumping-reach height. Models were constructed for estimates on Trials 1 and 10, with actor height and maximum jumping-reach height as factors. A model including participant height accounted for a significant amount of variance for self-estimates on Trial 1 $\left(r^{2}=.64\right)[F(1,18)=31.80, p<.01]$ and on Trial 10 $\left(r^{2}=.53\right)[F(1,18)=20.22, p<.01]$. Entering maximum jumping-reach into the model accounted for substantially more variance on both Trial $1\left(r^{2}=.85\right)[F(2,17)=46.84$, $p<.01]$ and Trial $10\left(r^{2}=.80\right)[F(2,17)=39.58, p<$ $.01]$. For the actor estimates, a model including the actor's height accounted for a significant amount of variance for Trial 10 only $\left(r^{2}=.38\right)[F(1,18)=10.91, p<.01]$, and

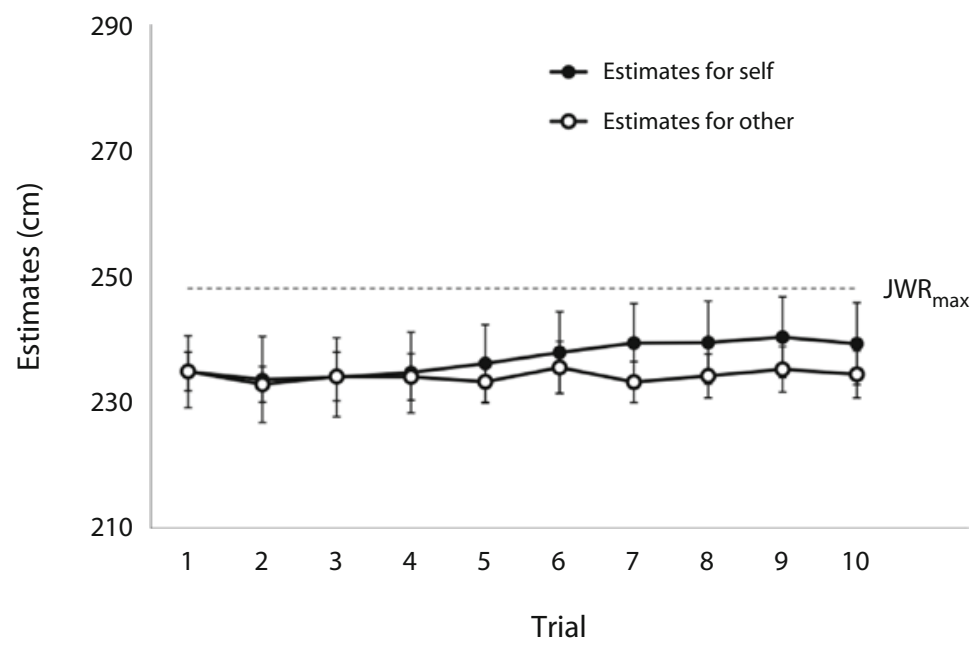

Figure 2. Results of Experiment 1. Mean perceptual estimates of maximum jumping-reach height for the self and for the other participant as a function of trials. Analyses showed that self-judgments increased significantly over trials; specifically, Trials 2 and 3 were significantly different from Trials 7-10. For the actor judgments, there was no significant trial effect. The dashed line at the top of the plot represents the actual mean maximum jumping-reach height $\left(J_{W} R_{\text {max }}\right)$ across all participants (each participant acted as both self and other, so the same line indicates $J_{W} R_{\text {max }}$ for self-estimates and actor estimates). Error bars correspond to one standard error. 
including the actor's maximum jumping-reach height did not account for any more variance.

The changes in self-estimates were apparent by Trial 4 and appear to have leveled off by Trial 7, at which point there remained an approximately $8.66-\mathrm{cm}$ underestimation of maximum jumping-reach height. The improved accuracy over trials for the self-judgments could reflect a process of attunement (the perceivers may have settled on a different variable by Trial 7 than they used on Trial 1) or perhaps, instead, a process of calibration, whereby the perceptual reports were anchored in the same variable but scaled differently. It is not possible to differentiate these possibilities using the present data. However, consistent with the interpretation of a change in variable use, the results of the regression analyses revealed that by the final trial, the self-estimates were more strongly correlated with actual jumping-reach height and less strongly correlated with participant height.

Estimates for the actor fluctuated somewhat but did not change systematically over trials. By Trial 10, those estimates were modestly correlated with the actor's actual maximum jumping-reach height, but the hierarchical regression results suggest that this may have been carried by the correlation of actor height and jumping-reach height. Although the self-estimates and actor estimates were initially similar, the difference in how they varied over time suggests some degree of independence of the ability to perceive affordances for another person from the ability to perceive affordances for the self, a result broadly consistent with other studies (Fischer, 2003, 2005; Ramenzoni et al., 2008b). A similar pattern of change in self-estimates and actor estimates over trials would have suggested a strong dependence of actor affordance estimates on the perceivers' own (actual or perceived) action capabilities. The results indicate otherwise, however. The absence of changes over trials in estimates for the actor suggests the relative independence of estimates for the actor from the learning processes (whether attunement, calibration, or a combination) that led to changes in self-estimates.

Although body sway or some other exploratory process might have provided the participants with the opportunity to improve estimates for themselves (cf. Mark et al., 1990; Stoffregen et al., 2005), any such processes did not lead to changes in the participants' estimates of maximum jumping-reach height for the other person. In Experiment 2, we determined whether participants' estimates of maximum jumping-reach height for an actor changed if the observers were allowed to watch an actor perform a task that was functionally related (but not identical) to jumping: squatting to lift a weight. Squatting is related to jumping inasmuch as it requires generation of vertical ground reaction force through contractions of the musculature of the legs. We asked whether watching an actor perform a task that shared this functional requirement with jumping would enable the observers to more accurately gauge the actor's action capabilities, relative to watching the actor perform a different task (rotating about the torso), which did not share the same functional requirements as producing a maximal vertical jump (cf. Ramenzoni et al., 2008).

\section{EXPERIMENT 2}

During the period of time over which estimates were collected in Experiment 1, the perceivers were apparently unable to discover an informational variable, or a new mapping from variable to perceptual report, that supported improved accuracy of perceiving an affordance for another. In contrast, the participants exhibited increased accuracy in perceiving an affordance for themselves. Providing explicit feedback to perceivers about the accuracy of their estimates for an actor would likely result in improved performance, but that might reflect the perceivers' ability to use explicit feedback more so than their ability to learn to prospectively perceive the limits of an agent's action capabilities.

Previous findings have demonstrated that the kinematics of an actor's movements are sufficient to inform observers about that actor's capabilities (Ramenzoni et al., 2008; Stoffregen et al., 1999). According to the kinematic specification of dynamics (KSD) principle (Runeson, 1977; Runeson \& Frykholm, 1983; Runeson, Juslin, \& Olsson, 2000), the kinematics and dynamics of an event are specific to one another (i.e., they relate in a one-toone fashion), because the forces and masses lawfully determine the resulting motion according to Newton's laws. From this principle of specificity, it follows that information related to the underlying dynamics of an event (or action) may be available in the kinematics of the event. Moreover, the salience of the kinematic information has been shown to influence perceptual accuracy. Bingham (1987) showed participants point-light displays of the kinematics of actors curling a handheld weight. Although the participants' perceptual reports scaled proportionally to the actual weight in all cases, when the kinematics were degraded by excluding joints in the display, the participants' ability to distinguish differing amounts of weight also decreased. The kinematics of a person's movement patterns - the patterns of displacement, velocity, and acceleration of the limbs and body segments - thus may be informative about the person's dynamic action capabilities, such as the person's capacity to produce force for jumping. How high a person can jump is determined by an actor's ability to produce appropriately patterned vertical force impulses relative to the actor's mass. On the basis of these considerations, we hypothesized that providing perceivers with information about a person's dynamic (i.e., force production) capabilities - even in the context of a behavioral (lifting/squatting) task that differed from the behavior about which the perceivers were to make judgments (jumping) — would enable the perceivers to more accurately gauge the actor's action capabilities and to improve their estimates of what is afforded that actor.

Perceivers initially provided two sets of estimates before watching the actor perform the lifting task. Then they watched the actor repeatedly lift (squat) $10 \%$ of his body mass. We expected that observing the actor perform the lifting task could inform the observers about the actor's ability to produce force with the legs in order to raise a 
mass (Dowling \& Vamos, 1993) and would thereby enable the perceivers to improve the accuracy of their perceptual estimates of the actor's maximum jumping-reach height. The estimates provided for the actor before and after watching the actor lift the mass were compared with estimates provided by a group of control participants who observed the actor perform a task that did not share underlying dynamics with jumping - standing while rotating the torso (i.e., twisting side-to-side about the waist). The participants in both groups also provided repeated estimates of their own maximum jumping-reach height.

On the basis of the results of Experiment 1, we expected to observe a significant improvement in perceptual estimates over time for self-estimates for both the experimental group and the control group. We furthermore expected the actor estimates to become more accurate for the experimental group after the observers watched the actor perform the lifting task but not to change for the control group.

\section{Method}

Participants. Thirty participants, ranging in height from 151.5 to $196 \mathrm{~cm}(M=170.6 \pm 11.12 \mathrm{~cm})$ and in maximum jumping-reach height from 220.2 to $298 \mathrm{~cm}(M=252.86 \pm 21.58 \mathrm{~cm})$ were asked to make maximum jumping-reach estimates for themselves and an actor. The actor, who was $180 \mathrm{~cm}$ tall, weighed $77.27 \mathrm{~kg}$, and had a maximum standing reach of $215.1 \mathrm{~cm}$ and a maximum jumpingreach height of $280.2 \mathrm{~cm}$, was the same for all of the participants. The participants were randomly assigned to each group ( $n=15$ for each group). There was a significant positive correlation between participant height and maximum jumping-reach height for each group (experimental, $r=.84, p<.05$; control, $r=.86, p<.05$ ) and a significant correlation between height and vertical jump for the control $(r=.54, p<.05)$ but not for the experimental group $(r=.46, p>.05)$.
Apparatus. The apparatus from Experiment 1 was used along with a 134-cm-long, 11-cm-diameter tube containing weights equaling $10 \%$ of the actor's body mass $(8 \mathrm{~kg})$.

Procedure. The procedures for the second experiment closely followed those in Experiment 1. The participants made estimates about the maximum limit of their own vertical jumping reach, as well as that of the actor. Estimates were again obtained using the method of limits and, as with Experiment 1, consecutive ascending-descending estimates were averaged together as one trial. Eight total experimental trials were presented in alternating blocks of two successive trials for the self and two successive trials for the actor. The order of judgment type conditions (self vs. actor) was counterbalanced across blocks and across participants.

At the midway point (after Trial 4), the experimental and control groups watched the actor perform the lifting or twisting task, respectively. Both the participant and the actor were led into an adjoining room. The participant and actor faced one another, standing $2 \mathrm{~m}$ apart. The participant was instructed to watch the actor perform either the squatting or the torso-rotation task, depending on the group assignment for the participant. In the control condition, the participant observed the actor, who stood rotating his torso from one side to another a total of 15 times. The rotation task did not involve any flexion or extension about the ankle, knee, or hip: The actor stood upright, with his legs straight, and twisted about the waist while holding his hands at his waist. In the experimental condition, the participant observed the actor lifting a tube, using a standard squat weight-lifting technique. The actor held the tube at chest level with both hands. While holding the tube, the actor lowered his torso by bending at the knees and keeping his back straight until his knees were flexed approximately $90^{\circ}$ and his thighs were parallel to the floor; at this point the actor stopped and raised himself back to a standing position. The actor lifted the tube in this manner a total of 15 times. Just prior to observing the actor, the participants were given the opportunity to haptically perceive the tube's mass by lifting it once in a similar manner. At the completion of the actor's task, both the participant and the actor returned to the other room and completed the remaining trials.

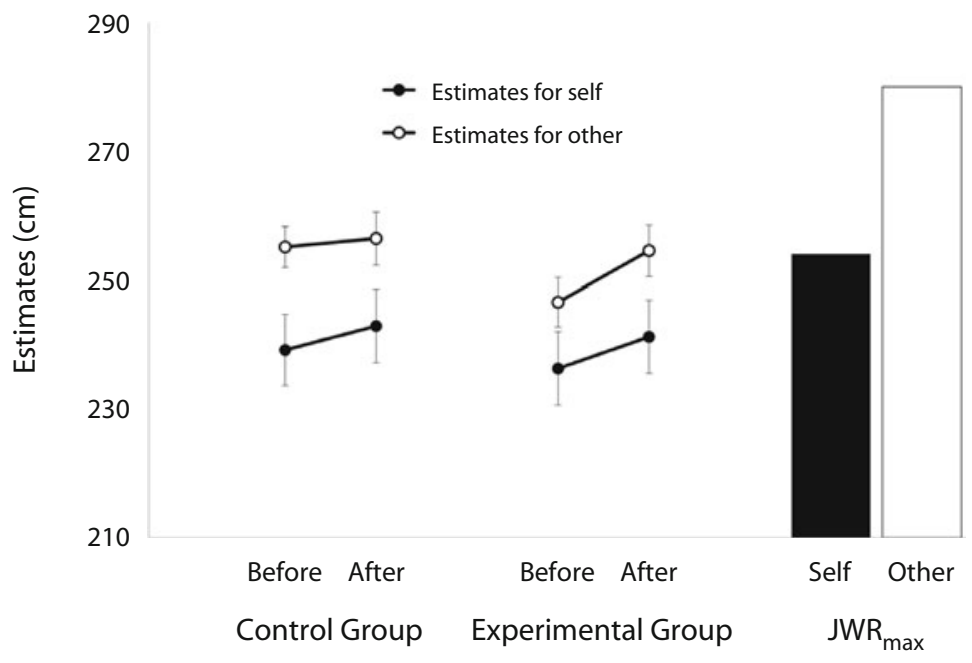

Figure 3. Results of Experiment 2. Mean perceptual estimates of maximum jumping-reach height for the observer and for the other actor as a function of block (before and after watching the actor perform either the twisting or the lifting task) for the control and experimental groups. The bars at the right represent the actual mean maximum jumping-reach height $\left(J W R_{\max }\right)$ for the participants (depicted in black) and the actor (depicted in white). Results indicate a significant block effect for self-estimates in both groups, but a significant block effect was observed for actor estimates only in the experimental group. Error bars correspond to one standard error. 


\section{Results and Discussion}

Trial estimates for each participant were averaged to yield one mean estimate per condition (self vs. other, before vs. after viewing the actor) per participant for both the experimental group and the control group (see Figure 3). Averaged across viewing blocks, the control group exhibited a tendency to underestimate maximum jumping-reach height for themselves (mean estimate $=$ $241.06 \pm 21.20 \mathrm{~cm}$; mean error $=$ mean estimate - actual jumping-reach height $=-13.34 \pm 13.47 \mathrm{~cm}$ ) and for the actor $($ mean estimate $=255.76 \pm 13.87 \mathrm{~cm}$; mean error $=$ $-24.44 \pm 13.87 \mathrm{~cm}$ ). A similar tendency was found for the experimental group in their self-estimates (mean estimate $=238.78 \pm 21.58 \mathrm{~cm}$; mean error $=-17.70 \pm$ $11.59 \mathrm{~cm}$ ) and those for the actor (mean estimate $=$ $250.56 \pm 15.28 \mathrm{~cm}$; mean error $=-29.64 \pm 15.28 \mathrm{~cm}$ ).

An ANOVA was conducted on the estimates with judgment (self vs. other) and block (first block-before watching the actor - vs. second block - after watching the actor) as within-subjects factors and group (experimental vs. control) as a between-subjects factor. The analysis revealed significant main effects of judgment $[F(1,28)=21.93, p<$ $\left..05, \eta_{\mathrm{p}}^{2}=.44\right]$ and block $\left[F(1,28)=27.92, p<.05, \eta_{\mathrm{p}}^{2}=\right.$ $.50]$. The group $\times$ block $\left[F(1,28)=6.80, p<.05, \eta_{\mathrm{p}}^{2}=\right.$ $.20]$ and the judgment $\times$ block $\times$ group $[F(1,28)=5.19$, $p<.05, \eta_{\mathrm{p}}^{2}=.16$ ] interactions were also significant.

Follow-up ANOVAs were performed to further investigate the significant three-way interaction. An ANOVA was performed on the data separately for each judgment type. For the self-estimates, there were significantly larger (and, consequently, more accurate) estimates for the second block than for the first block $[F(1,28)=11.28, p<$ $\left..05, \eta_{\mathrm{p}}^{2}=.29\right]$, regardless of group. An analysis of the estimates provided for the actor revealed a main effect of block $\left[F(1,28)=15.16, p<.05, \eta_{\mathrm{p}}^{2}=.35\right]$ and also a significant block $\times$ group interaction $[F(1,28)=6.31, p<$ $\left..05, \eta_{\mathrm{p}}^{2}=.18\right]$. The interaction was driven by the experimental group participants providing significantly larger estimates for the actor in the second block than in the first block $[t(14)=-5.95, p<.05]$. No difference between blocks was found for the estimates for the actor provided by the control group $(p>.05){ }^{3}$

The results showed that the participants' self-estimates of maximum jumping-reach height became more accurate in the second block than they were in the first block. Such an increase in accuracy, which was observed for the participants in both the control group and the experimental group, was expected on the basis of the results of Experiment $1 .{ }^{4}$ The control group's estimates for the actor's maximum jumping-reach height did not change across blocks, also similar to the findings of Experiment 1 . However, the experimental group's estimates were more accurate in the second block, after they had observed the actor performing the lifting task, than in the first block. Watching the actor perform a task related to the dynamics of jumping helped the perceivers improve the accuracy of their estimates of the actor's jumping-reach capabilities. Watching the actor perform an unrelated action (the torso-rotation task witnessed by the control group) did not help the participants gauge the actor's jumping-reach capabilities.
These results support the idea that individuals may be able to acquire knowledge about another person from the other person's movement patterns. However, this ability seems to be limited to the case of observing movements that are functionally related to the task being judged. Kinematic patterns relating to one class of body movements can be informative about a nonidentical, but functionally similar, class of body movements. Although the specific force generation requirements of squatting and jumping are not identical, both tasks involve the capacity to generate vertical force by contracting the leg muscles and producing angular accelerations of body segments about the ankles, knees, and hips. These findings suggest that this similarity was sufficient to allow the participants to improve perceptual estimates (i.e., to learn) about the actor's capacity to perform a vertical jump (cf. Ramenzoni et al., 2008). Perceivers might thus gain the ability to prospectively determine the limits of a person's action capabilities by watching the person perform functionally related actions, without ever watching the person perform the exact action in question.

\section{GENERAL DISCUSSION}

The main focus of this study was on the estimates that the participants provided for the actor. Experiment 1 showed that those estimates, which were initially very close to the estimates provided by the participants for themselves, did not change over trials, in contrast to the participants' self-estimates, which increased significantly (and became more accurate) over trials. Consequently, the accuracy of the self-estimates and the actor estimates was quite different on the last trial. That pattern of results has two related implications.

First, the results speak to the relative independence of perceptual judgments of what is afforded the self versus the other. We did not measure or manipulate information independently of the material basis of the jumping-reach affordance in this study (a dissociation that would be required in order to make definitive claims about information usage). However, it appears that by the end of the experiment, if not even at the beginning, the participants relied on different sources of information when making the two types of judgments. For example, the participants may have relied on some relation between eye-height-scaled visual information (cf. Mark et al., 1990; Ramenzoni et al., 2008b) and proprioceptive information about force production capacity (Ramenzoni et al., 2008a) for making self-judgments and (visual) kinematic information (Ramenzoni et al., 2008) relative to proprioceptive information about their own force production capacity (Ramenzoni et al., 2008a) when making actor judgments. Alternatively, it is possible that over trials, the participants became differently calibrated to the same informational quantity and scaled their self-estimates versus actor estimates differently in response to the same perceptual variable(s). Future research must be directed at independently manipulating these potential information sources.

Regardless of the possible informational basis for the perceptual estimates, the results suggest a divergence 
between perceiving affordances for oneself and perceiving affordances for another person. Even if judgments of affordances for another person are affected by the perceiver's own action capabilities or situated relation to the environment, as has been suggested in previous studies (e.g., Ramenzoni et al., 2008a), the present results indicate that affordance judgments are not entirely dependent on or determined by attributes of the perceiver. If that were the case, the self-judgments and the actor judgments would have changed over trials in the same fashion. This finding is inconsistent with embodied simulation models that claim a strong dependency on the observer's own action capabilities (e.g., Gallese, 2005).

The second implication of the results of Experiment 1 is the possibility that there was information for learning (Jacobs \& Michaels, 2007) that enabled the perceivers to improve estimates for themselves, but such information was either not available or may have gone undetected or simply unused with regard to modifying estimates for the actor. As it was conceived by Jacobs and Michaels (2007) in their direct learning approach, information for learning refers to variables that specify how to reduce perceptual error or nonoptimality of perception-action. Postural sway or other exploratory movements of the eyes or head (J. J. Gibson, 1966) were apparently sufficient in the case of self-estimates to allow the perceivers to improve the accuracy of their responses (Mark et al., 1990; Stoffregen et al., 2005) but were apparently insufficient in the case of estimates for the actor. Because the perceivers in Experiment 1 did not show spontaneous improvement in their estimates for the actor, in Experiment 2, we attempted to provide a means of inducing perceptual learning about the action capabilities of the actor. This was accomplished by allowing the perceivers to view the actor perform a task that shared its underlying dynamics with jumping (i.e., producing vertically directed force through lowerlimb muscular contractions). The results of the present study contribute to the recently introduced direct learning approach by demonstrating that explicit feedback about the accuracy of reports may not be required for learning. Simply providing perceivers with a salient source of new information may help the perceivers improve their perceptual estimates. Once again, however, our claims about information must be qualified, because we did not independently manipulate the informational and material bases for the maximum jumping-reach affordance.

\section{Kinematic Specification of Action-Scaled Affordances for an Actor}

Jumping and reaching is a dynamic movement that depends largely on the forces that an actor is able to generate at a particular time. It could be argued that the veridical perception of maximum jumping-reach height requires the perceiver to have knowledge related to these dynamic properties. For the self, perception is continuously a multimodal affair. That is, information in the optic array is available concurrently with other types of information (e.g., proprioceptive information about body mass and dimensions, sensations related to muscular exertion or fatigue, etc.) that provide participants with knowledge about their dynamic action capabilities. Perceivers may become sensitive to patterns of stimulation across different energetic (i.e., optical and mechanical) media - to patterns in the global array (Stoffregen \& Bardy, 2001) - that relate to how high they can jump.

In contrast, viewing an actor who is simply standing does not seem to reveal in any obvious way the underlying dynamic properties of that actor. In Experiment 2, we found that providing observers with opportunities to view the actor exercising his dynamic action capabilities could help the perceivers provide more accurate perceptual reports of the boundary between what was reachable by the actor via a jump and what was not. The manipulation in Experiment 2 was motivated by findings that observers are able to acquire knowledge about the underlying dynamics (forces, mass, momentum exchange) of an event (Kim, Turvey, \& Carello, 1993; Runeson \& Vedeler, 1993) on the basis of kinematic visual information. For instance, observers are able to accurately estimate the weight of a hefted object, as well as the amount of effort exerted by the actor performing the activity, on the basis of a point-light display of an actor lifting the object (Bingham, 1987; see also Runeson \& Frykholm, 1983; Shim, Carlton, \& Kim, 2004). This ability to perceive dynamics from purely kinematic displays is explained as a consequence of the lawful, causal relation of dynamics to kinematics - the KSD principle (Runeson, 1977; Runeson \& Frykholm, 1983).

Along with the KSD principle, other findings demonstrate that information gained from one activity can improve the perception of one's action capabilities for another activity with related dynamics (Oudejans, Michaels, Bakker, \& Dolné, 1996). Together, these considerations led us to hypothesize that perceivers might benefit from watching an actor engage in movements related (but not necessarily identical) to the target action when making judgments for others (Ramenzoni et al., 2008). That is, kinematic patterns associated with one class of body movements (squatting/ lifting) may inform perceivers about another, nonidentical class of body movements (jumping), if the movements share the same underlying dynamic capacities. In contrast, observing a movement that did not depend critically on the capacity to produce vertical force impulses with the legs (twisting the torso) was not expected to support improved perceptual accuracy. As was hypothesized, when the participants viewed the actor performing the dynamically related squatting/lifting task, their estimates of the actor's maximum jumping-reach height improved, but watching the actor perform the twisting task did not improve the estimates. Our findings do not speak to whether the perceivers became attuned to a variable specific to jumping capacity or to a more general variable that captures the vertical force production capacity of an actor (i.e., leg strength), which could map to more than one affordance (e.g., maximum jumping-reach height and maximum weight that can be lifted by squatting). In any case, however, the data demonstrate that the salience of the variable in question (general vertical force production capacity or jumping capacity) is a function of the type of movement of the actor, suggesting that for perceiving maximum jumping-reach height, the kinematics of squatting were more useful than the 
kinematics of torso rotation for enabling the perceivers to gauge the actor's maximum jumping-reach height.

\section{Perceiving Maximum \\ Jumping-Reach Height for Oneself}

In both experiments, the participants' initial perceptual reports underestimated their own maximum jumping-reach height. The accuracy of the perceivers' estimates for themselves improved with repeated opportunities to provide perceptual reports, however, even in the absence of explicit feedback about the accuracy of the reports. In Mark et al. (1990) and Stoffregen et al. (2005), similar changes in affordance judgments (of whether a chair afforded sitting) were observed for participants whose action capabilities had been modified. The changes in their participants' affordance judgments were interpreted as reflecting processes of attunement and/or calibration: The participants had to adjust to their newly modified action capabilities and were capable of doing so without feedback, presumably because postural sway produced transformations of stimulus arrays that revealed new information about what the environment afforded. Interestingly, however, in both of those studies, a control group whose action capabilities were not modified did not exhibit changes in affordance judgments over time, in contrast to the results of the present study.

The contrast in those results might be due to the participants' having more daily experience sitting on objects and chairs of different heights than they have at jumping to reach objects suspended overhead. Although it is reasonable to assume that our participants had some prior experience jumping to reach objects, it is likely that they had less experience doing so than choosing to sit on chairs, stools, tables, or other surfaces of different heights. Moreover, our experimental setup likely introduced unique environmental and task constraints, such as not allowing the participants to take a running or walking approach before jumping, or the perceptual reporting method itself. The observed pattern of improvements in self-judgments may thus have reflected learning to meet the unique constraints of the task at hand.

\section{Implications for Social Perception-Action}

Theories of social perception-action and cognition may benefit from a better understanding of the relation between the neurocognitive mechanisms hypothesized to operate in these behaviors and the perceptual activity necessary to support the functioning of those mechanisms. Toward that end, it is important to gain a better understanding of the perceptual information used in determining whether an action is possible for an agent. If motor resonance in the mirror neuron system plays an important role in social cognition and perception-action (e.g., Gallese, Keysers, \& Rizzolatti, 2004), it is important to understand how information serves as a medium that makes this resonance possible.

The present results are consistent with information-based approaches to social perception-action (Ramenzoni et al., 2008b). Greater accuracy of the estimates for the actor could be achieved in the absence of explicit feedback by allowing the perceiver to observe the actor perform a functionally related but nonidentical action. Observed changes in the accuracy of judgments for the self did not appear to have a direct influence on judgments for the actor; instead, both types of judgment appeared to rely on distinct processes. Individuals were able to become more accurate in perceiving what an environment afforded another only when they observed the actor perform an action related to jumping.

These findings may also provide some insight as to the factors that might constrain simulation mechanisms. According to embodied simulation advocates (e.g., Gallese, 2005; Gallese \& Goldman, 1998), motor simulations are the result of mapping the actions of another person to the perceiver's own neural capacity to produce those actions. One theoretical implication of this view is that perceptions of another person's (past, present, and possible future) actions are tightly bound to one's own action capabilities. In Experiment 1, this view would predict that perceptual judgments for the self and the other exhibit similar patterns over time. That is, changes in judgments concerning the action capabilities of the self would influence and would thus be accompanied by similar changes in perceptual judgments concerning another. The present results were not consistent with that prediction. They suggest, instead, that in order for simulation mechanisms to function adaptively and accurately, they must not only have online access to concurrent visual information (Fischer, 2005; Ramenzoni et al., 2008b), but the observer must be attuned and calibrated to the right action-relevant visual parameters related to the agent's action capabilities.

\section{AUTHOR NOTE}

This research was supported by NSF Grants BCS 0716319, BCS 0728743, and BCS 0926662. V.C.R. is now at the Department of Psychology, University of Virginia. Correspondence concerning this article should be addressed to M. A. Riley, Department of Psychology, 4150C Edwards 1, ML 0376, University of Cincinnati, Cincinnati, OH 45221 0376 (e-mail: michael.riley@uc.edu).

\section{REFERENCES}

Bingham, G. P. (1987). Kinematic form and scaling: Further investigations on the visual perception of lifted weight. Journal of Experimental Psychology: Human Perception \& Performance, 13, 155-177.

Dowling, J. J., \& Vamos, L. (1993). Identification of kinetic and temporal factors related to vertical jump performance. Journal of Applied Biomechanics, 9, 95-110.

FAJEN, B. R. (2005a). Calibration, information, and control strategies for braking to avoid a collision. Journal of Experimental Psychology: Human Perception \& Performance, 31, 480-501.

FAJEN, B. R. (2005b). Perceiving possibilities for action: On the necessity of calibration and perceptual learning for the visual guidance of action. Perception, 34, 717-740.

FAJEN, B. R. (2005c). The scaling of information to action in visually guided braking. Journal of Experimental Psychology: Human Perception \& Performance, 31, 1107-1123.

FAJEN, B. R., \& Devaney, M. C. (2006). Learning to control collisions: The role of perceptual attunement and action boundaries. Journal of Experimental Psychology: Human Perception \& Performance, 32, 300-313.

FISCHER, M. H. (2003). Can we correctly perceive the reaching range of others? British Journal of Psychology, 94, 487-500.

FisCHER, M. H. (2005). Action simulation for others is not constrained by one's own postures. Neuropsychologia, 43, 28-34.

Gallese, V. (2005). Embodied simulation: From neurons to phenomenal experience. Phenomenology \& the Cognitive Sciences, 4, 23-48.

Gallese, V., \& Goldman, A. (1998). Mirror neurons and the simulation theory of mind-reading. Trends in Cognitive Sciences, 12, 493-501. 
Gallese, V., Keysers, C., \& Rizzolatti, G. (2004). A unifying view of the basis of social cognition. Trends in Cognitive Sciences, 8, 396403 .

Gibson, E. J. (1991). An odyssey in learning and perception. Cambridge, MA: MIT Press.

Gibson, J. J. (1966). The senses considered as perceptual systems. Boston: Houghton Mifflin.

GiBson, J. J. (1979). The ecological approach to visual perception. Boston: Houghton Mifflin.

Guilford, J. P. (1954). Psychometric methods. New York: McGraw-Hill.

Hove, P., Riley, M. A., \& Shockley, K. (2006). Perceiving affordances of hockey sticks by dynamic touch. Ecological Psychology, 18, 163189.

JaCOBS, D. M., \& Michaels, C. F. (2006). Lateral interception I: Operative optical variables, attunement, and calibration. Journal of Experimental Psychology: Human Perception \& Performance, 32, 443-458.

Jacobs, D. M., \& Michaels, C. F. (2007). Direct learning. Ecological Psychology, 19, 321-349.

Kim, N.-G., Turvey, M. T., \& CArello, C. (1993). Optical information about the severity of upcoming contacts. Journal of Experimental Psychology: Human Perception \& Performance, 19, 179-193.

Knoblich, G., \& Sebanz, N. (2008). Evolving intentions for social interaction: From entrainment to joint action. Philosophical Transactions of the Royal Society B, 363, 2021-2031.

Lamm, C., Fischer, M. H., \& Decety, J. (2007). Predicting the actions of others taps into one's own somatosensory representations-A functional MRI study. Neuropsychologia, 45, 2480-2491.

MARK, L. S. (2007). Perceiving the actions of other people. Ecological Psychology, 19, 107-136.

Mark, L. S., Balliett, J. A., Craver, K. D., Douglas, S. D., \& Fox, T. (1990). What an actor must do in order to perceive the affordance for sitting. Ecological Psychology, 2, 325-366.

Oudejans, R. R. D., Michaels, C. F., Bakker, F. C., \& Dolné, M. A. (1996). The relevance of action in perceiving affordances: Perception of catchableness of fly balls. Journal of Experimental Psychology: Human Perception \& Performance, 22, 879-891.

PACHerie, E., \& Dokic, J. (2006). From mirror neurons to joint actions. Cognitive Systems Research, 7, 101-112.

Ramenzoni, V. C., Riley, M. A., Davis, T., Shockley, K., \& ArmSTRONG, R. (2008). Tuning in to another person's action capabilities: Perceiving maximal jumping-reach height from walking kinematics. Journal of Experimental Psychology: Human Perception \& Performance, 34, 919-928.

Ramenzoni, V. C., Riley, M. A., Davis, T., \& Snyder, J. (2005). Perceiving whether or not another person can use a step to reach an object. In H. Heft \& K. L. Marsh (Eds.), Studies in perception and action VIII (pp. 15-18). Mahwah, NJ: Erlbaum.

Ramenzoni, V. C., Riley, M. A., Shockley, K., \& Davis, T. (2008a). Carrying the height of the world on your ankles: Encumbering observers reduces estimates of how high an actor can jump. Quarterly Journal of Experimental Psychology, 61, 1487-1495.

Ramenzoni, V. C., Riley, M. A., Shockley, K., \& Davis, T. (2008b). An information-based approach to action understanding. Cognition, 106, 1059-1070.

RUNESON, S. (1977). On the possibility of "smart" perceptual mechanisms. Scandinavian Journal of Psychology, 18, 172-179.

Runeson, S., \& Frykholm, G. (1983). Kinematic specification of dynamics as an informational basis for person-and-action perception: Expectation, gender recognition, and deceptive intention. Journal of Experimental Psychology: General, 112, 585-615.
Runeson, S., Juslin, P., \& Olsson, H. (2000). Visual perception of dynamic properties: Cue heuristics versus direct-perceptual competence. Psychological Review, 107, 525-555.

Runeson, S., \& Vedeler, D. (1993). The indispensability of precollision kinematics in the visual perception of relative mass. Perception \& Psychophysics, 53, 617-632.

Sebanz, N., Bekkering, H., \& Knoblich, G. (2006). Joint action: Bodies and minds moving together. Trends in Cognitive Sciences, 10, 70-76.

Shim, J., Carlton, L. G., \& KIM, J. (2004). Estimation of lifted weight and produced effort through perception of point-light display. Perception, 33, 277-291.

Stoffregen, T. A., \& Bardy, B. G. (2001). On specification and the senses. Behavioral \& Brain Sciences, 24, 195-261.

Stoffregen, T. A., Gorday, K. M., Sheng, Y.-Y., \& Flynn, S. B. (1999). Perceiving affordances for another person's actions. Journal of Experimental Psychology: Human Perception \& Performance, 25, 120-136.

Stoffregen, T. A., YAng, C.-M., \& Bardy, B. G. (2005). Affordance judgments and nonlocomotor body movement. Ecological Psychology, 17, 75-104.

WEAST, J. A. (2009). Expert sensitivity to kinematic information in perception of affordances for others. Unpublished master's thesis, University of Cincinnati, Cincinnati, $\mathrm{OH}$.

Withagen, R., \& Michaels, C. F. (2005). The role of feedback information for calibration and attunement in perceiving length by dynamic touch. Journal of Experimental Psychology: Human Perception \& Performance, 31, 1379-1390.

\section{NOTES}

1. This was done to reduce the possibility of an order effect. Subsequent analyses revealed no such effect; estimates provided by the participants who served first as the observer did not differ from those provided by the participants who served first as the actor.

2. For both experiments, an analysis of the perceptual error (mean estimate - actual jumping-reach height) yielded a pattern of results identical to that of an analysis of the mean perceptual estimates.

3. In Experiment 1, we conducted an extensive series of correlation analyses to identify relations among estimates, participant height, and maximum jumping-reach height. In Experiment 2, we were unable to perform those analyses for the actor estimates, because the same person always served as the actor. It was possible to perform those analyses on the self-estimates, and when submitted to similar hierarchical models, the overall pattern of correlations in Experiment 2 was largely consistent with the results of the self-estimate correlations in Experiment 1. However, because those results were not central to Experiment 2, we do not report them in detail here.

4. For the sake of comparison with Experiment 2, we reanalyzed the data from Experiment 1, collapsing across Trials 1-5 and Trials 6-10, comparing the averages of perceptual reports across those two blocks of trials. Like the control group results in Experiment 2, in Experiment 1 there was a significant main effect of trial block $[F(1,19)=5.66$, $\left.p<.05, \eta_{\mathrm{p}}^{2}=.23\right]$ and a significant block $\times$ estimate type interaction $\left[F(1,19)=13.46, p<.05, \eta_{\mathrm{p}}^{2}=.41\right]$, driven by a change across blocks in self-estimates but no change in actor estimates.

(Manuscript received April 6, 2009; revision accepted for publication December 3, 2009.) 